\title{
Negative Differential Resistance in Bidirectional Threshold Switching of Ag/HfOx/Pt Device
}

\author{
zhiguo jiang \\ Institute of Semiconductors, South China Normal University \\ Dongliang Wang \\ Institute of Semiconductors, South China Normal University \\ Yan Li \\ Institute of Semiconductors, South China Normal University \\ Yong Zhang \\ Institute of Semiconductors, South China Normal University \\ Xinman Chen ( $\nabla$ xmchenscnu@163.com ) \\ South China Normal University
}

\section{Nano Express}

Keywords: Negative Differential Resistance, Threshold Switching, Conductive Filaments

Posted Date: February 17th, 2020

DOI: https://doi.org/10.21203/rs.2.23747/v1

License: (c) (i) This work is licensed under a Creative Commons Attribution 4.0 International License. Read Full License 


\title{
Negative Differential Resistance in Bidirectional Threshold Switching of $\mathrm{Ag} / \mathrm{HfO}_{\mathrm{x}} / \mathrm{Pt}$ Device
}

\author{
Zhiguo Jiang ${ }^{a}$, Dongliang Wang ${ }^{a}$, Yan Li ${ }^{a}$, Yong Zhang ${ }^{a}$, Nasir Khisro ${ }^{b}$, Xinman Chen ${ }^{a, *}$ \\ ${ }^{a}$ Guangdong Engineering Research Center of Optoelectronic Functional Materials and Devices, \\ Institute of Semiconductor Science and Technology, South China Normal University, Guangzhou \\ 510631, China \\ ${ }^{b}$ Physics Department, University of Kotli Azad Kashmir Pakistan, Azad Kashmir 11100, Pakistan
}

Abstract

In this work, the dependence of negative differential resistance (NDR) on compliance current $\left(I_{c c}\right)$ was investigated based on $\mathrm{Ag} / \mathrm{HfO}_{\mathrm{x}} / \mathrm{Pt}$ resistive memory device. Tunable conversion from bidirectional threshold switching (TS) to memory switching (MS) were achieved through enhancing $I_{c c}$. NDR can be observed in TS as $I_{c c}$ is below $800 \mu \mathrm{A}$ but vanishes in MS. The switching voltages and readout windows of TS evolve with $I_{c c}$. Furthermore, the dynamic conductance $(d I / d V)$ as a function of time in NDR can be well illustrated by capacitor-like relaxation equation, and the relaxation time constant is significantly dependent on $I_{c c}$. These phenomena were elucidated from viewpoint of nanofilament evolution controlled by $I_{c c}$ as well as nanocapacitor effects originated from nanofilament gap. The $I_{c c}$-dependent NDR as well as conversion between $\mathrm{TS}$ and $\mathrm{MS}$ on $\mathrm{Ag} / \mathrm{HfO}_{\mathrm{x}} / \mathrm{Pt}$ resistive memory device indicates its potential application as a multifunctional electronic device.

\section{Keywords:}

RRAM, Negative Differential Resistance, Threshold Switching, Conductive Filaments.

* Author to whom correspondence should be addressed: xmchenscnu@163.com; 


\section{Introduction}

Resistance random accessory memory (RRAM) based on the resistive switching (RS) phenomenon in capacitor-like metal-insulator-metal (MIM) devices has attracted significant attention due to its simple structure, easy fabrication, fast switching speed and superior scaling property. ${ }^{[1-3]}$ Two basic switching modes were generally included according to the electrical switching behaviors of RRAM, namely memory switching (MS) and threshold switching (TS). ${ }^{[4-6]}$ The MS is a kind of nonvolatile behavior, both high resistance state (HRS) and low resistance state (LRS) can be maintained after removing the external bias, enabling the application in the nonvolatile memories, reconfigurable logic and neuromorphic computing, which has been intensively investigated in various transitional metal oxides, such as $\mathrm{HfO}_{2},{ }^{[7]} \mathrm{Al}_{2} \mathrm{O}_{3},{ }^{[8]} \mathrm{SrTiO}_{3},{ }^{[9]}$ and so on. On the contrary, the TS is a type of volatile behavior with an S-shape I-V curve that works in limited voltage range between two critical values called the holding voltage $\left(\mathrm{V}_{\mathrm{H}}\right)$ and threshold voltage $\left(\mathrm{V}_{\mathrm{TH}}\right)$. Differing from nonvolatile MS, LRS in TS will switch back to HRS as applied bias is below $\mathrm{V}_{\mathrm{H}}$, that is, HRS retains below $\mathrm{V}_{\mathrm{H}}$ and only LRS is displayed above $\mathrm{V}_{\mathrm{TH}}$. Many investigations have demonstrated the excellent selector function of TS to suppress the sneak problem of high-density crossbar arrays as connected with RRAM in series [10-13]. Even conversion between MS and TS under the suitable external excitation was also reported. ${ }^{[5,6,14,15]}$

Lately, an important nonlinear carrier-transport phenomenon-negative differential resistance (NDR), referring to a decrease in current with increasing 
voltage or vice versa, has been discovered accompanying with various RS, such as unipolar $\mathrm{RS}$ on $\mathrm{Ag} / \mathrm{SiO}_{2} / \mathrm{Pt}$ device, ${ }^{[16]}$ multilevel $\mathrm{RS}$ on $\mathrm{Pt} / \mathrm{BaSrTiO}_{3} / \mathrm{SrTiO}_{3}: \mathrm{Nb}$ device, ${ }^{[17]}$ bipolar $\mathrm{RS}$ on $\mathrm{Ag} / \mathrm{ZnO} / \mathrm{Zn}$ device ${ }^{[18]}$ and flexible organic memristive memory ${ }^{[19]}$. However, the origin of NDR in RS is controversial. Discharging electrons from metal nanocrystals, charge storage processes between interfaces, separation of oxygen vacancies and trapped electrons, and different work functions of materials have been proposed to contribute to NDR. Furthermore, the compliance current $\left(I_{c c}\right)$ is a vital parameter for RRAM to understand and manipulate RS, but the effects of $I_{c c}$ on NDR are still insufficient. In this work, conversion from bidirectional TS to MS were achieved on $\mathrm{Ag} / \mathrm{HfO}_{\mathrm{x}} / \mathrm{Pt}$ device through controlling $I_{c c}$. NDR can be observed in TS with $I_{c c}$ lower than $800 \mu \mathrm{A}$. The dependence of NDR on $I_{c c}$ was also investigated by manipulating TS via $I_{c c}$. The phenomena can be elucidated in the view of conductive filaments model as well as nanocapacitor effects originated from filament gap.

\section{Experimental section}

The $\mathrm{HfO}_{\mathrm{x}}$ films were directly deposited on the available $\mathrm{Pt} / \mathrm{TiO}_{2} / \mathrm{SiO}_{2} / \mathrm{Si}$ substrates by atomic layer deposition (ALD) system with tetrakis-(dimethylamido)-hafnium $\left(\mathrm{Hf}\left(\mathrm{NMe}_{2}\right)_{4}\right)$ as the precursor source, purified water as the oxygen source and purified nitrogen as the carrier gas. For one typical growth cycle, $\mathrm{Hf}\left(\mathrm{NMe}_{2}\right)_{4} / \mathrm{N}_{2} / \mathrm{H}_{2} \mathrm{O} / \mathrm{N}_{2}$ with the exposure time of $0.15 \mathrm{~s} / 15 \mathrm{~s} / 0.15 \mathrm{~s} / 15 \mathrm{~s}$ was performed, and 250 cycles were carried out to deposit $\mathrm{HfO}_{\mathrm{x}}$ thin film with thickness of $\sim 30 \mathrm{~nm}$. To fabricate $\mathrm{Ag} / \mathrm{HfO}_{\mathrm{x}} / \mathrm{Pt}$ devices, the top $\mathrm{Ag}$ electrodes were subsequently 
deposited on the surface of as-fabricated $\mathrm{HfO}_{\mathrm{x}}$ films through a shadow mask. The diameter and the thickness of the top electrodes are $\sim 100 \mu \mathrm{m}$ and $\sim 100 \mathrm{~nm}$, respectively. The schematic diagram of $\mathrm{Ag} / \mathrm{HfO}_{\mathrm{x}} / \mathrm{Pt}$ devices was illustrated as inset of

Fig. 1(a). The electrical performances of device were characterized by scanning the current-voltage (I-V) curves using a Keithley 4200 semiconductor parameter analyzer at room temperature. The voltage step is fixed to be $0.05 \mathrm{~V}$, and the collecting time for each data point is $0.1 \mathrm{~ms}$ containing delay time and holding time. For all measurements, the bias voltage was applied on the bottom Pt electrodes, while the top Ag electrodes were grounded all along.

\section{Results and discussion}

A pristine $\mathrm{Ag} / \mathrm{HfO}_{\mathrm{x}} / \mathrm{Pt}$ device stays $\mathrm{HRS}$. To get the switching effects, an electroforming process was generally needed. It has been reported that the resistive switching performances are well dependent on the electroforming process like polarity, sequence of $I_{c c .}{ }^{[20-21]}$ Also, double and asymmetric two-step electroforming are reported to be beneficial to stabilize the resistive switching. ${ }^{[7]}$ Here, an incomplete electroforming process with $I_{c c}$ of $10 \mu \mathrm{A}$ was applied by performing a positive sweeping $0 \sim+8 \mathrm{~V}$. As Fig. 1(a) indicates, the current is far lower than $10 \mu \mathrm{A}$ and there is no abrupt current rise in this process. After that, the sweeping setup of $0 \mathrm{~V} \rightarrow-0.4 \mathrm{~V} \rightarrow 0 \mathrm{~V} \rightarrow 0.4 \mathrm{~V} \rightarrow 0 \mathrm{~V}$ was subsequently performed. Fig. 1(b) exhibits I-V curves with $I_{c c}$ of $10 \mu \mathrm{A}$ in semi-logarithmic scale. It is evident that a Set switching from HRS to LRS firstly occurs at a negative threshold voltage $\left(\mathrm{V}_{\mathrm{TH}^{-}}\right) \sim-0.25 \mathrm{~V}$ in the following sweeping from $0 \mathrm{~V}$ to $-0.4 \mathrm{~V}$, and a Reset switching from LRS to HRS 
appears at negative hold voltage $\left(\mathrm{V}_{\mathrm{H}^{-}}\right) \sim-0.12 \mathrm{~V}$ as voltage reverted back from $-0.4 \mathrm{~V}$ to $0 \mathrm{~V}$. Similarly, the nearly symmetric Set and Reset switching occur in the positive bias branch with a positive threshold voltage $\left(\mathrm{V}_{\mathrm{TH}^{+}}{ }^{+}\right) \sim 0.22 \mathrm{~V}$ and hold voltage $\left(\mathrm{V}_{\mathrm{H}}^{+}\right) \sim$ $0.05 \mathrm{~V}$, respectively. It is worthy of noting that when voltage scanned backward from $+0.4 \mathrm{~V} /-0.4 \mathrm{~V}$ to $0 \mathrm{~V}$, NDR occurred obviously once voltage is below $\mathrm{V}_{\mathrm{H}}$ in both negative and positive bias branches. Fig. 1(c) and Fig. 1(d) display the typical I-V and dynamic conductance $(d I / d V)-V$ curves at linear scale where NDR appears. Whether the voltage sweeps from $\mathrm{V}_{\mathrm{H}}{ }^{+}$or $\mathrm{V}_{\mathrm{H}}{ }^{-}$to $0 \mathrm{~V}$, current increases gradually, while dynamic conductance degrades. Similar NDR was achieved in all TS curves. Moreover, it is of significant importance to emphasize that negative current was detected in positive NDR bias but positive current emerges in negative NDR bias.

In spite of facts that conversion between TS and MS depending on $I_{c c}$ as well as NDR have been widely reported, ${ }^{[16-18,22-24]} I_{c c}$-dependent NDR is still undiscovered. For this sake, the bidirectional TS with NDR was further purchased and systematically compared under various $I_{c c}$ of $1 \mu \mathrm{A}, 10 \mu \mathrm{A}, 20 \mu \mathrm{A}, 50 \mu \mathrm{A}, 100 \mu \mathrm{A}, 150 \mu \mathrm{A}$, $500 \mu \mathrm{A}$ and $800 \mu \mathrm{A}$. Fig. 2(a) and 2(b) show some typical I-V curves and corresponding evolution of switching voltages with $I_{c c}$, including $\mathrm{V}_{\mathrm{H}}{ }^{+}, \mathrm{V}_{\mathrm{H}^{-}}, \mathrm{V}_{\mathrm{TH}^{+}}{ }^{+}$and $\mathrm{V}_{\mathrm{TH}}{ }^{-}$. As Fig. 2(a) indicates, $\mathrm{Ag} / \mathrm{HfO}_{\mathrm{x}} / \mathrm{Pt}$ device evidently manifests the bidirectional TS with NDR as $I_{c c}$ is below $800 \mu \mathrm{A}$. With enhancing $I_{c c}$ from $1 \mu \mathrm{A}$ to $800 \mu \mathrm{A}$, all threshold voltages $\mathrm{V}_{\mathrm{TH}^{+}}{ }^{+}$and $\mathrm{V}_{\mathrm{TH}^{-}}$evolve towards the lower value (Fig. 2(b)). Although the holding voltage $\mathrm{V}_{\mathrm{H}}{ }^{+}$and $\mathrm{V}_{\mathrm{H}^{-}}$increase slightly in small $I_{c c}$, both descend eventually as $I_{c c}$ rises up. Meanwhile, the readout windows, defined as the voltage gap 
between $\mathrm{V}_{\mathrm{H}}$ and $\mathrm{V}_{\mathrm{TH}}$, drop gradually with increasing $I_{c c}$ (Inset in Fig. 2(b)). However, TS of $\mathrm{Ag} / \mathrm{HfO}_{\mathrm{x}} / \mathrm{Pt}$ device becomes unstable when $I_{c c}$ is over $800 \mu \mathrm{A}$, and converses to MS as $I_{c c}$ is larger than $1 \mathrm{~mA}$. Fig. 2(c) displays I-V curves within continuous 600 cycles with $I_{c c}$ of $5 \mathrm{~mA}$, featuring with typical nonvolatile MS. The corresponding evolution of Reset/Set voltages and resistance at 0.05V, shown in Fig. 2(d), indicate that the uniform $\mathrm{RS}$ can be obtained on $\mathrm{Ag} / \mathrm{HfO}_{\mathrm{x}} / \mathrm{Pt}$ device in a suitable $I_{c c}$. Interestingly, NDR which emerges in all bidirectional TS, cannot be observed in MS performances.

As result of the evolution of TS with $I_{c c}$, the unique dependence of NDR on $I_{c c}$ can be probably explored. During the voltage scanning, the voltage step is fixed to be $0.05 \mathrm{~V}$, and the collecting time for each data point is calculated to be $0.1 \mathrm{~ms}$ containing delay time and holding time. Therefore, $d I / d V$ as a function of time in NDR region was derived from various $I_{c c}$ to further understand the effects of $I_{c c}$ on NDR. Fig. 3(a) 3(d) illustrate the typical $d I / d V$-t plots with $I_{c c}$ of $1 \mu \mathrm{A}, 10 \mu \mathrm{A}, 20 \mu \mathrm{A}, 50 \mu \mathrm{A}$, respectively, which were derived from negative NDR bias region. The black lines are fitting results with an exponential relaxation equation $d I / d V=A \exp \left(-\frac{t}{\tau}\right)$, where $t$ is the relaxation time, $A$ refers to the initial value of $d I / d V$, and $\tau$ is the relaxation time constant. Obviously, the experimental data is in good accordance with the fitting results, which means $d I / d V$ - $t$ plot can be expressed by a capacitor-like relaxation equation. It also suggests that NDR in TS here is possibly originated from capacitive effects. Fig. 3(e) displays the evolution of parameter $\tau$ with investigated $I_{c c}$ from $1 \mu \mathrm{A}$ to $800 \mu \mathrm{A}$. Interestingly, there are two stages for variation of $\tau$ with $I_{c c} . \tau$ decreases 
slightly from $\sim 0.18 \mathrm{~ms}$ to $\sim 0.16 \mathrm{~ms}$ as $I_{c c}$ changes from $1 \mu \mathrm{A}$ to $50 \mu \mathrm{A}$ but with small deviation in each $I_{c c}$. Then, $\tau$ rises dramatically to be $\sim 0.26 \mathrm{~ms}$ with large deviation as $I_{c c}$ exceeds $100 \mu \mathrm{A}$. It implies the deteriorated uniformity of TS on $\mathrm{Ag} / \mathrm{HfO}_{\mathrm{x}} / \mathrm{Pt}$ device in large $I_{c c}$.

Many investigations have directly observed growth and annihilation of $\mathrm{Ag}$ nanofilaments via electrochemical redox process in Ag-based programmable metallization cells that causes Set and Reset switching in MS. ${ }^{[25,26]}$ The tiny Ag nanofilaments, especially in a low $I_{c c}$, are not stable due to Rayleigh instability. Therefore, the tiny nanofilaments will be dissolved spontaneously under surface energy as well as the diffusion into matrix due to the concentration gradient and thermal effects, ${ }^{[26,27]}$ resulting in the volatile TS accordingly. For both MS and TS, $I_{c c}$ plays a particularly important role in manipulating nanofilaments morphology. ${ }^{[16,18,25-}$ 27] Therefore, on basis of abovementioned analysis, the $I_{c c}$-dependent NDR in this work can be understood from viewpoint of the evolution of Ag conductive nanofilaments coupling with the capacitive effects of nanocapacitor resulted from nanofilaments gap. Fig. 4 schematically illustrates the overall evolution of $\mathrm{Ag}$ nanofilaments and nanocapacitor as well as the corresponding equivalent circuits in TS and MS. Initially, incomplete electroforming did not switch $\mathrm{Ag} / \mathrm{HfO}_{\mathrm{x}} / \mathrm{Pt}$ device to LRS. As the negative bias was applied on the Pt electrode, Ag nanofilaments grew along the old one and switched device to LRS as bias came to $\mathrm{V}_{\mathrm{TH}^{-}}$(Fig. 4(a)). But tiny nanofilaments formed due to the limitation of low $I_{c c}$. The tiny Ag nanofilaments can be equivalently considered as a resistance and are unstable in nano scale, which 
dissolved spontaneously under surface energy, resulting in switching back to HRS at $\mathrm{V}_{\mathrm{H}^{-}}$(Fig. 4(b)). Then, the residual nanofilaments in $\mathrm{HfO}_{\mathrm{x}}$ films were disconnected with nanogap. Due to high conductivity of Ag nanofilaments, the overall device resembles nanocapacitor with two resistances in series, the equivalent circuit is shown in right side of Fig. 4(b). As the applied bias was below $\mathrm{V}_{\mathrm{H}^{-}}$, the nanocapacitor discharged, enhancing current of circuit, namely NDR. It also created the reverse current owing to the opposite electric field direction between nanocapacitor and applied bias. Similar processes occurred in the positive bias region, as shown in Fig. 4(c) and 4(d).

According to the model of a plane-parallel capacitor, the capacitance $(C)$ can be expressed as $C=\frac{\varepsilon S}{4 \pi k d}$, where $\varepsilon$ is dielectric constant of dielectrics, $S$ is area of parallel conductive nanofilaments cross sectional area, $k$ is electrostatic constant, $d$ is gap between counter nanofilaments. As mentioned, it is the discharging processes of nanocapacitor as bias is below hold voltage $\left(\mathrm{V}_{\mathrm{H}}\right)$ that generates NDR phenomenon in $\mathrm{Ag} / \mathrm{HfO}_{\mathrm{x}} / \mathrm{Pt}$ device and reverse current in the circuit. This discharging current of nanocapacitor can be characterized by equation of $I_{t}=I_{s} \exp \left(-\frac{t}{\tau}\right)$, where $I_{s}$ is saturate current of pristine nanocapacitor. $\tau$ is the relaxation time constant referring to $R C$, where $R$ is resistance value of residual nanofilaments. So, $d I$ is also exponential function with time, but $d V$ is constant due to the fixed voltage steps in measurement, which results in exponential $d I / d V-t$ plot. And the relaxation time constant $\tau$ correlates with $R C$, where both $R$ and $C$ vary with $I_{c c}$. As $I_{c c}$ increases from $1 \mu \mathrm{A}$ to $50 \mu \mathrm{A}, R$ decreases because of robust nanofilaments, leading to the 
lower $\tau$. However, $S$ widens and $d$ shortens as result of growth of nanofilaments under larger $I_{c c}$. Both can cause the larger $C$, resulting in dramatical enhancement of $\tau$ accordingly, shown as Fig. 3(e). As $I_{c c}$ further increases, the Ag nanoparticles inside filaments can further grow and connect with each other eventually to form continuous nanofilaments, which require more surface energy to dissolve due to large diameter. It causes disappearance of TS and dominance of MS, in which reversible formation and rupture of the robust nanofilaments results in Set and Reset switching, shown as Fig. 4(e) and 4(f). However, NDR in TS disappears in MS. This is reasonably believed that the slight current discharged from nanocapacitor is negligible compared with that of LRS in MS. In any case, the $I_{c c}$-dependent NDR as well as conversion between TS and MS on $\mathrm{Ag} / \mathrm{HfO}_{\mathrm{x}} / \mathrm{Pt}$ resistive memory device indicate its potential application as a multifunctional electronic device.

\section{Conclusions}

In summary, we investigated the dependence of $\mathrm{NDR}$ on $I_{c c}$ based on $\mathrm{Ag} / \mathrm{HfO} \mathrm{X}_{\mathrm{x}} / \mathrm{Pt}$ resistive memory device. In addition to bipolar memory switching in large $I_{c c}$, the as-fabricated device exhibit bidirectional threshold switching with NDR in low $I_{c c}$. This NDR is significantly dependent on $I_{c c}$, and the dynamic conductance $d I / d V$ - $t$ plot in NDR is good accordance to the capacitor-like relaxation equation. These phenomena were attentively elucidated from viewpoint of filaments evolution controlled by $I_{c c}$ as well as nanocapacitor effects resulted from filaments gap. 


\section{Conflicts of interest}

There are no conflicts to declare.

\section{Acknowledgements}

The authors gratefully acknowledge support from the National Nature Science Foundation of China (61674059), the Science and Technology Planning Project of Guangdong Province (2015A010103012), the Science and Technology Planning Project of Guangzhou City (201804010399), and the Innovative Project of Education Department of Guangdong Province (2017KTSCX050). 


\section{References}

[1] Waser R, Aono M (2009) Nanoionics-based resistive switching memories. Nanoscience and Technology: A Collection of Reviews from Nature Journal S 158-165. https://doi.org/10.1142/9789814287005_0016

[2] Yang JJ, Pickett MD, Li X, et al (2008) Memristive switching mechanism for metal/oxide/metal nanodevices. Nature Nanotechnology 3:429-433. https: //doi.org/10.1038/nnano.2008.160

[3] Kagan CR, Lifshitz E, Sargent EH, Talapin D V. (2016) Building devices f rom colloidal quantum dots. Science 353:. https://doi.org/10.1126/science.aac $\underline{5523}$

[4] Watanabe Y, Bednorz JG, Bietsch A, et al (2001) Current-driven insulator-c onductor transition and nonvolatile memory in chromium-doped $\mathrm{SrTiO}_{3}$ sing le crystals. Applied Physics Letters 78:3738-3740. https://doi.org/10.1063/1.1 $\underline{377617}$

[5] Chang SH, Lee JS, Chae SC, et al (2009) Occurrence of both unipolar me mory and threshold resistance switching in a NiO film. Physical Review L etters 102:1-4. https://doi.org/10.1103/PhysRevLett.102.026801

[6] Peng HY, Li YF, Lin WN, et al (2012) Deterministic conversion between memory and threshold resistive switching via tuning the strong electron cor relation. Scientific Reports 2:1-6. https://doi.org/10.1038/srep00442

[7] Chen $\mathrm{X}, \mathrm{Hu} \mathrm{W}, \mathrm{Li} \mathrm{Y}$, et al (2016) Complementary resistive switching beha viors evolved from bipolar $\mathrm{TiN} / \mathrm{HfO}_{2} / \mathrm{Pt}$ device. Applied Physics Letters 10 
8:. https://doi.org/10.1063/1.4941287

[8] Hubbard WA, Kerelsky A, Jasmin G, et al (2015) Nanofilament Formation and Regeneration during $\mathrm{Cu} / \mathrm{Al}_{2} \mathrm{O}_{3}$ Resistive Memory Switching. Nano Lette rs 15:3983-3987. https://doi.org/10.1021/acs.nanolett.5b00901

[9] Mikheev E, Hoskins BD, Strukov DB, Stemmer S (2014) Resistive switchi ng and its suppression in $\mathrm{Pt} / \mathrm{Nb}: \mathrm{SrTiO}_{3}$ junctions. Nature Communications 5: . https://doi.org/10.1038/ncomms4990

[10] Liu D, Cheng H, Wang G, et al (2013) Diode-like volatile resistive switc hing properties in amorphous Sr-doped $\mathrm{LaMnO}_{3}$ thin films under lower cur rent compliance. Journal of Applied Physics 114:1-6. https://doi.org/10.1063/ $\underline{1.4826362}$

[11] Liu T, Verma M, Kang Y, Orlowski M (2012) Volatile resistive switching in $\mathrm{Cu} / \mathrm{TaO} \mathrm{x} / \delta-\mathrm{Cu} / \mathrm{Pt}$ devices. Applied Physics Letters 101:2-6. https://doi.o $\underline{\mathrm{rg} / 10.1063 / 1.4746276}$

[12] Chen W, Barnaby HJ, Kozicki MN (2016) Volatile and Non-Volatile Switc hing in $\mathrm{Cu}-\mathrm{SiO}_{2}$ Programmable Metallization Cells. IEEE Electron Device Letters 37:580-583. https://doi.org/10.1109/LED.2016.2540361

[13] Li Y, Yuan P, Fu L, et al (2015) Coexistence of diode-like volatile and multilevel nonvolatile resistive switching in a $\mathrm{ZrO}_{2} / \mathrm{TiO}_{2}$ stack structure. $\mathrm{Na}$ notechnology 26: https://doi.org/10.1088/0957-4484/26/39/391001

[14] Qi J, Olmedo M, Zheng JG, Liu J (2013) Multimode resistive switching i n single $\mathrm{ZnO}$ nanoisland system. Scientific Reports 3:1-6. https://doi.org/10. 
$\underline{1038 / \text { srep02405 }}$

[15] Pan C, Ji Y, Xiao N, et al (2017) Coexistence of Grain-Boundaries-Assist ed Bipolar and Threshold Resistive Switching in Multilayer Hexagonal Bor on Nitride. Advanced Functional Materials 27: https://doi.org/10.1002/adfm. $\underline{201604811}$

[16] Sun H, Liu Q, Long S, et al (2014) Multilevel unipolar resistive switchin $\mathrm{g}$ with negative differential resistance effect in $\mathrm{Ag} / \mathrm{SiO} 2 / \mathrm{Pt}$ device. Journal of Applied Physics 116:1-6. https://doi.org/10.1063/1.4898807

[17] He J, Zhu J, Ma C, et al (2019) Negative differential resistance and mult ilevel resistive switching in $\mathrm{BaSrTiO}_{3}$ films. Applied Physics Letters 115:16. https://doi.org/10.1063/1.5113883

[18] Kadhim MS, Yang F, Sun B, et al (2018) A resistive switching memory device with a negative differential resistance at room temperature. Applied Physics Letters 113:1-6. https://doi.org/10.1063/1.5037191

[19] Zhu S, Sun B, Ranjan S, et al (2019) Mechanism analysis of a flexible o rganic memristive memory with capacitance effect and negative differential resistance state. APL Materials 7:. https://doi.org/10.1063/1.5100019

[20] Chen X, Hu W, Wu S, Bao D (2014) Stabilizing resistive switching perfo rmances of $\mathrm{TiN} / \mathrm{MgZnO} / \mathrm{ZnO} / \mathrm{Pt}$ heterostructure memory devices by program ming the proper compliance current. Applied Physics Letters 104:1-5. https: //doi.org/10.1063/1.4863744

[21] Li Y, Pan X, Zhang Y, Chen X (2015) Write-Once-Read-Many-Times and 
Bipolar Resistive Switching Characteristics of $\mathrm{TiN} / \mathrm{HfO}_{2} / \mathrm{Pt}$ Devices Depend ent on the Electroforming Polarity. IEEE Electron Device Letters 36:1149-1 152. https://doi.org/10.1109/LED.2015.2477421

[22] Pickett MD, Borghetti J, Yang JJ, et al (2011) Coexistence of memristanc e and negative differential resistance in a nanoscale metal-oxide-metal syste m. Advanced Materials 23:1730-1733. https://doi.org/10.1002/adma.20100449 $\underline{7}$

[23] Zhou G, Duan S, Li P, et al (2018) Coexistence of Negative Differential Resistance and Resistive Switching Memory at Room Temperature in TiOx Modulated by Moisture. Advanced Electronic Materials 4:1700567. https://doi.org/10.1002/aelm.201700567

[24] Du G, Li H, Mao Q, Ji Z (2016) Controllable volatile to nonvolatile resis tive switching conversion and conductive filaments engineering in $\mathrm{Cu} / \mathrm{ZrO}_{2} /$ Pt devices. Journal of Physics D: Applied Physics 49:. https://doi.org/10.108 $\underline{8 / 0022-3727 / 49 / 44 / 445105}$

[25] Sun H, Liu Q, Li C, et al (2014) Direct Observation of Conversion Betw een Threshold Switching and Memory Switching Induced by Conductive Fil ament Morphology. Advanced Functional Materials 24:5679-5686. https://doi. $\underline{\text { org/10.1002/adfm.201401304 }}$

[26] Hsiung CP, Liao HW, Gan JY, et al (2010) Formation and instability of s ilver nanofilament in Ag-based programmable metallization cells. ACS Nan o 4:5414-5420. https://doi.org/10.1021/nn1010667 
[27] Yang JJ, Strukov DB, Stewart DR (2013) Memristive devices for computi ng. Nature Nanotechnology 8:13-24. https://doi.org/10.1038/nnano.2012.240 


\section{Figure Captions}

Figure 1 (a) An incomplete electroforming process on $\mathrm{Ag} / \mathrm{HfO}_{\mathrm{x}} / \mathrm{Pt}$ device. Inset shows schematic diagram of $\mathrm{Ag} / \mathrm{HfO}_{\mathrm{x}} / \mathrm{Pt}$ device. (b) Typical I-V curves of bidirectional TS at $I_{c c}=10 \mu \mathrm{A}$. Typical $\mathrm{I}-\mathrm{V}$ and derived $d I / d V-V$ curves in NDR of positive (c) and negative (d) bias region with $I_{c c}=10 \mu \mathrm{A}$.

Figure 2 (a) Bidirectional TS characteristic curves at $I_{c c}$ of $1 \mu \mathrm{A}, 10 \mu \mathrm{A}, 150 \mu \mathrm{A}$, $800 \mu$ A. (b) Evolution of $\mathrm{V}_{\mathrm{H}^{+}}, \mathrm{V}_{\mathrm{H}^{-}}, \mathrm{V}_{\mathrm{TH}^{+}}{ }^{+}$and $\mathrm{V}_{\mathrm{TH}^{-}}{ }^{-}$with $I_{c c}$. Inset shows the corresponding evolution of readout windows. (c) Typical I-V curves of MS with $I_{c c}=5 \mathrm{~mA}$. (d) Evolution of resistance at $0.05 \mathrm{~V}$ and switching voltages for MS within 600 cycles.

Figure $3 d I / d V$ - $t$ plots and fitting results with relaxation equation for NDR with various $I_{c c}$ : (a) $1 \mu \mathrm{A}$, (b) $10 \mu \mathrm{A}$ (c) $20 \mu \mathrm{A}$ and (d) $50 \mu \mathrm{A}$. (e) Evolution of relaxation time constant $\tau$ with $I_{c c}$.

Figure 4 Schematic diagrams of the model corresponding to the various states of device. After a low $I_{c c}$ electroforming, tiny conductive nanofilaments formed and the device performs as TS: (a) Negative ON states. (b) Negative OFF states. (c) Positive OFF states. (d) Positive ON states. The device performs as MS in large $I_{c c}$ : (e) OFF states. (f) ON states. 
Figures
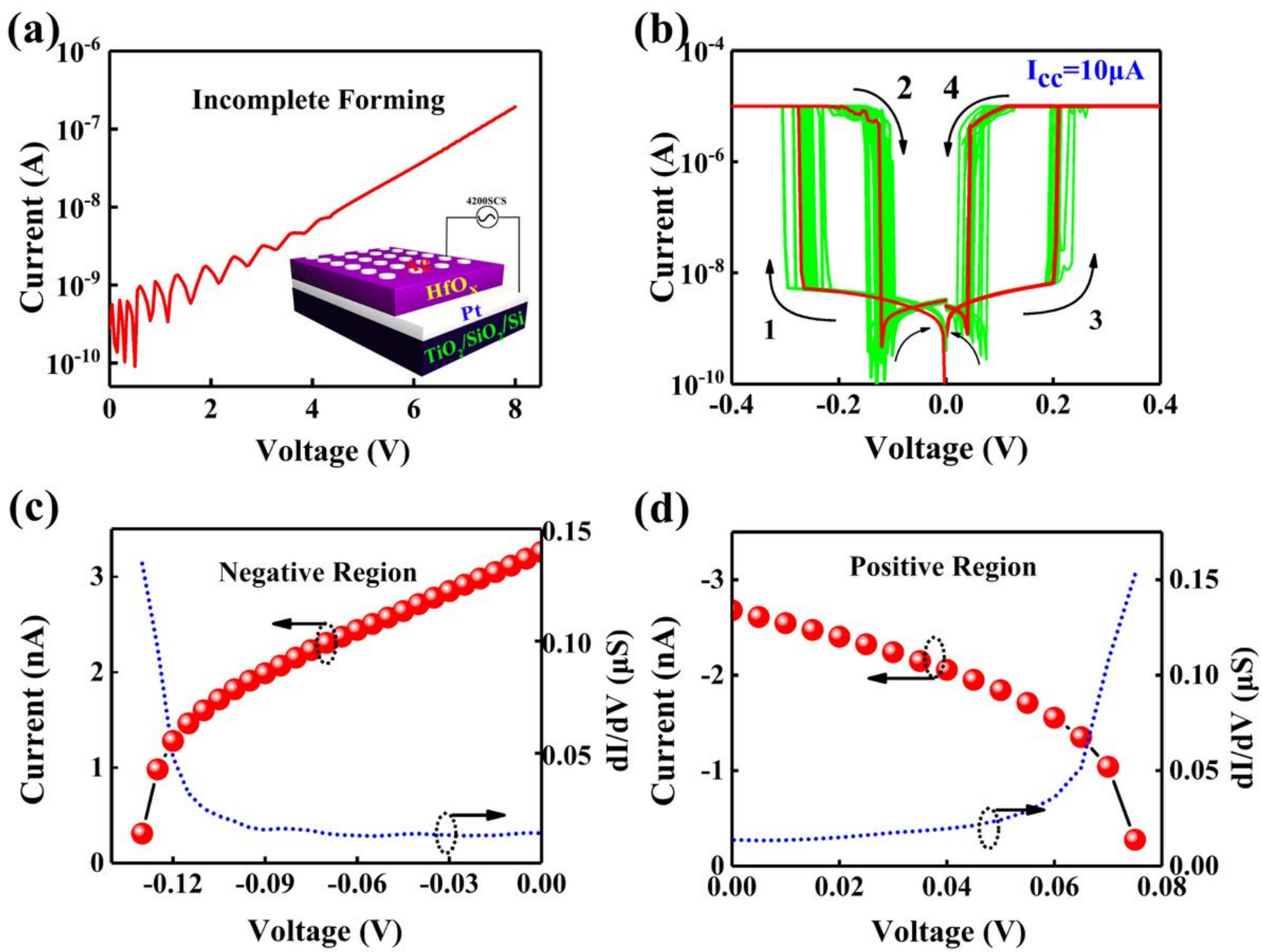

Figure 1

(a) An incomplete electroforming process on $\mathrm{Ag} / \mathrm{HfOx} / \mathrm{Pt}$ device. Inset shows schematic diagram of $\mathrm{Ag} / \mathrm{HfOx} / \mathrm{Pt}$ device. (b) Typical I V curves of bidirectional TS at Icc $=10 \mu \mathrm{A}$. Typical I V and derived dl/dV V curves in NDR of positive (c) and negative (d) bias region with Icc $=10 \mu \mathrm{A}$. 
(a)
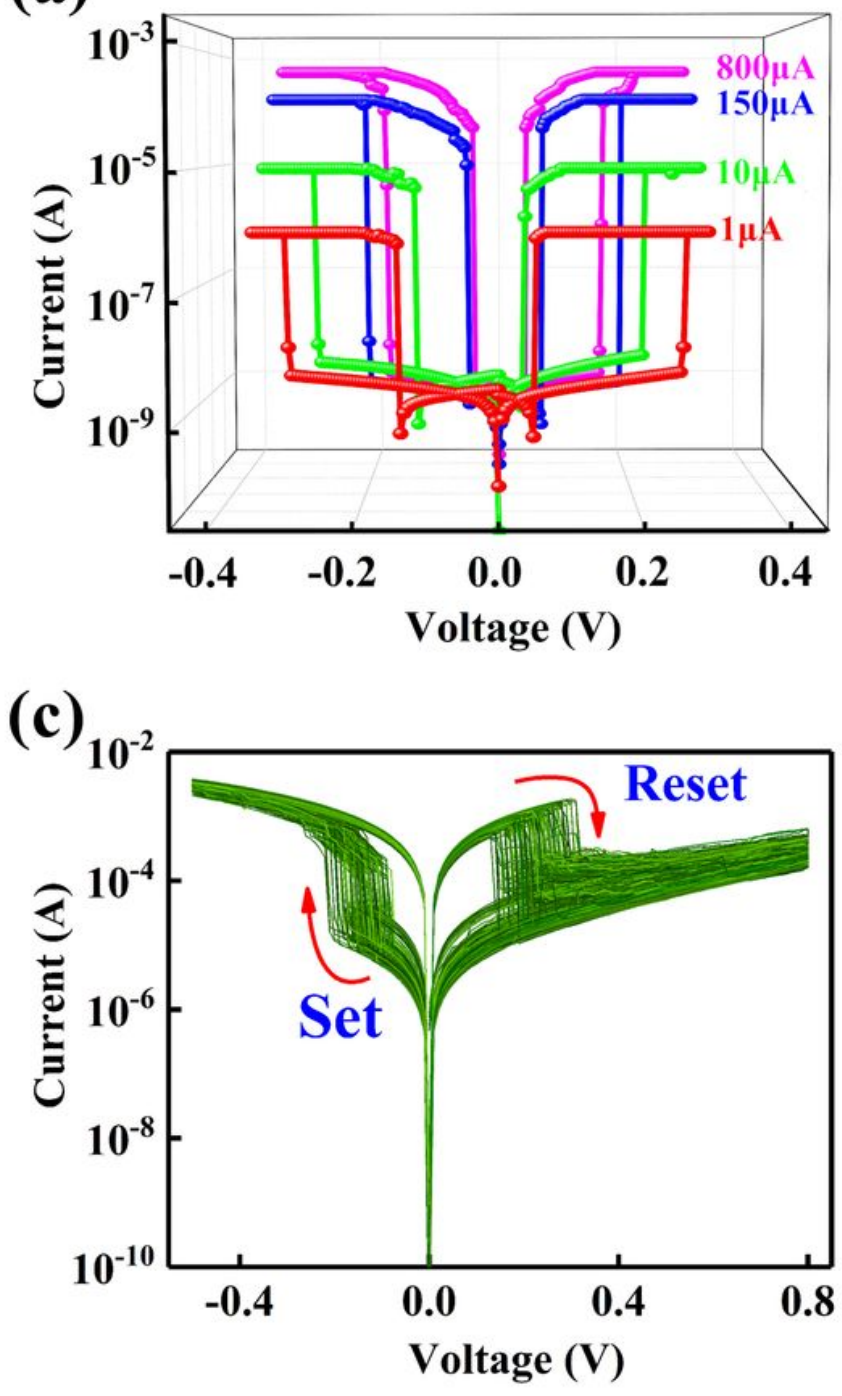

(b)
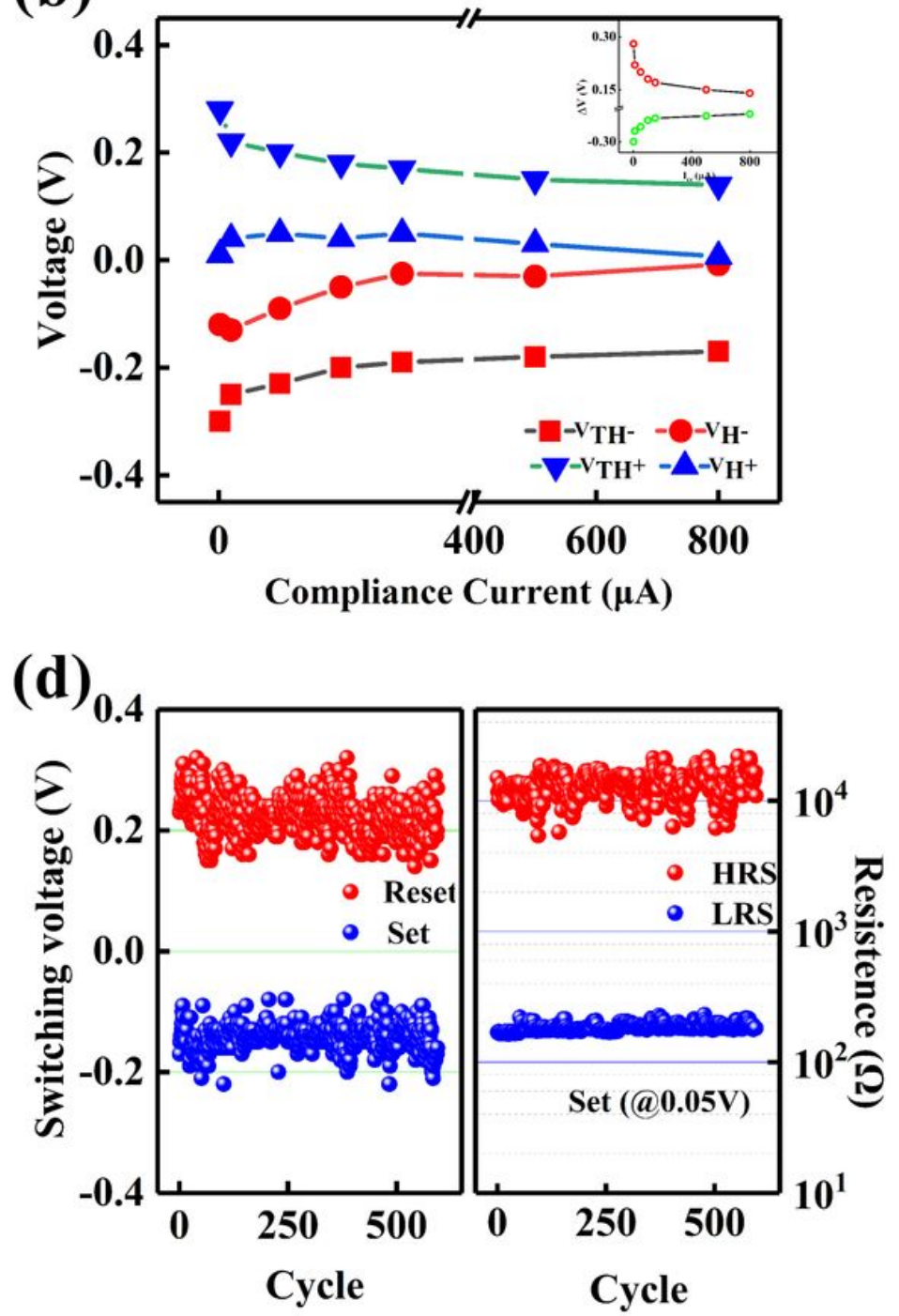

Figure 2

(a) Bidirectional TS characteristic curves at Icc of $1 \mu \mathrm{A}, 10 \mu \mathrm{A}, 150 \mu \mathrm{A}, 800 \mu \mathrm{A}$. (b) Evolution of $\mathrm{VH}+, \mathrm{VH}-$, VTH+ and VTH with Icc. Inset shows the corresponding evolution of readout windows. (c) Typical I V curves of MS with Icc=5 mA. (d) Evolution of resistance at $0.05 \mathrm{~V}$ and switching voltages for MS within 600 cycles. 

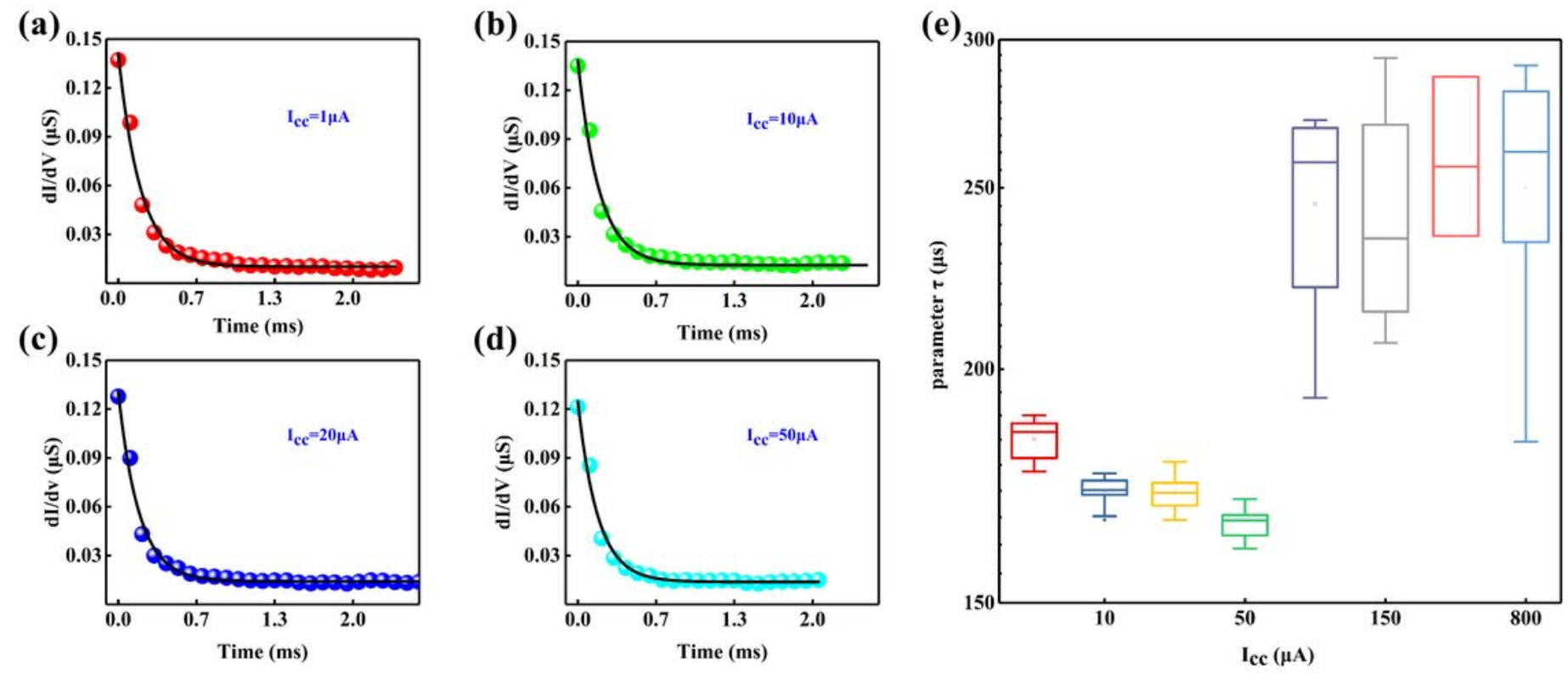

Figure 3

$\mathrm{dl} / \mathrm{dV} t$ plots and fitting results with relaxation equation for NDR with various Icc: (a) $1 \mu \mathrm{A}$, (b) $10 \mu \mathrm{A}$ (c) $20 \mu \mathrm{A}$ and (d) $50 \mu \mathrm{A}$. (e) Evolution of relaxation time constant $\otimes$ with Icc.

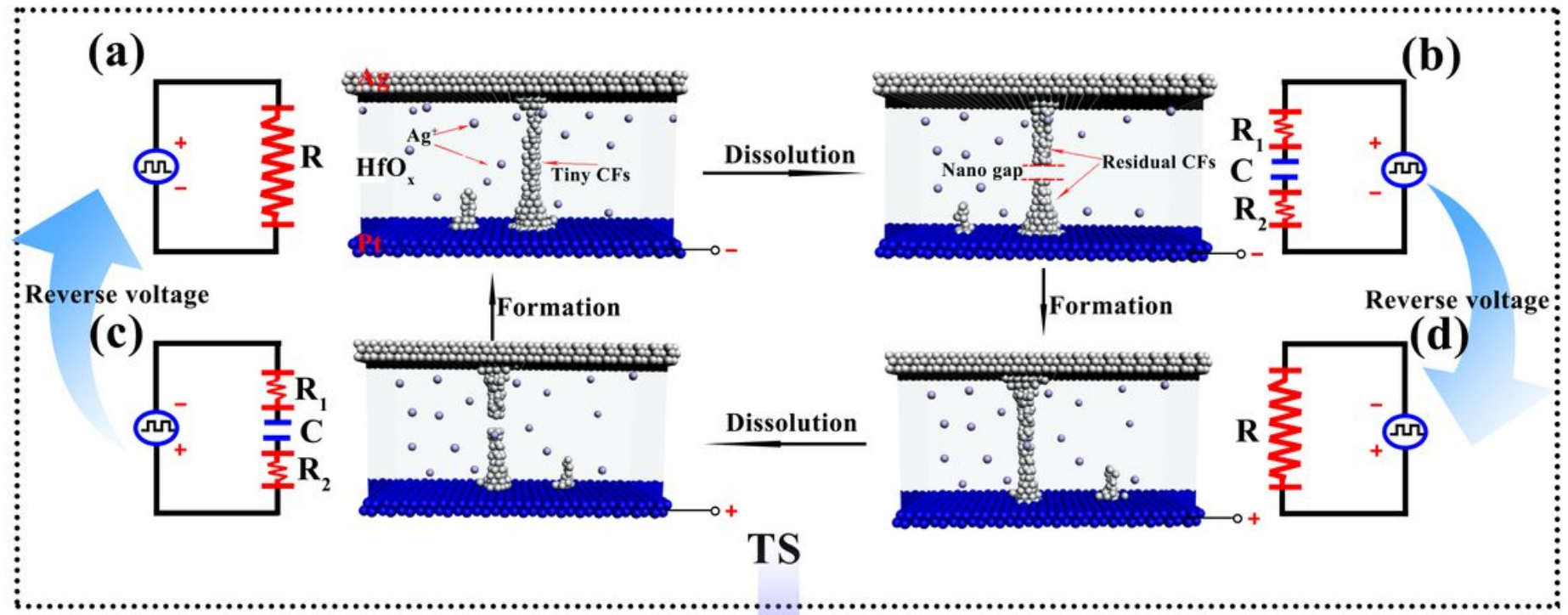

Increase

Compliance Current

(e)

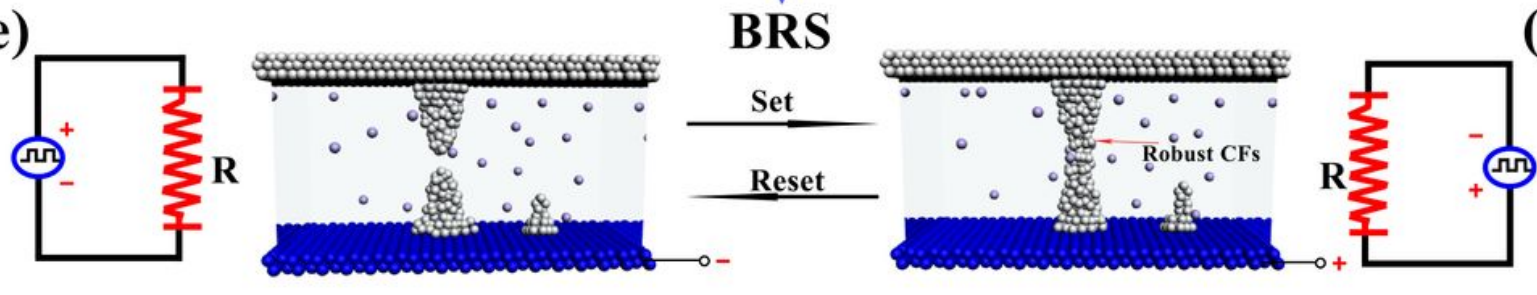




\section{Figure 4}

Schematic diagrams of the model corresponding to the various states of device. After a low Icc electroforming, tiny conductive nanofilaments formed and the device performs as TS: (a) Negative ON states. (b) Negative OFF states. (c) Positive OFF states. (d) Positive ON states. The device performs as MS in large Icc: (e) OFF states. (f) ON states. 